\title{
Biosimilars: To switch or not to switch - that is the question
}

\section{Zoltán Szekanecz}

Division of Rheumatology, Faculty of Medicine, University of Debrecen, Debrecen, Hungary

\section{Introduction}

There has been increasing financial pressure with respect to reimbursement of targeted therapies all over Europe. For example, in 2014, four out of the 10 bestselling biopharmaceuticals (adalimumab, infliximab, etanercept, rituximab) were used in the field of immune-mediated inflammatory diseases (IMID) including rheumatoid arthritis (RA), spondyloarthritides (SpA), psoriasis and inflammatory bowel diseases (IBD). The total sales of these four compounds increased from 8.2 billion USD in 2004 to 37.7 billion in 2014 [1]. Possibilities of reducing the cost would be unit price reduction, limitation of patient number or limitation of access to drugs [2]. Certainly it would be less ethical to officially limit access or the number of patients. Therefore, one needs to seek cheaper drugs.

Expiration of patent protection of several biologics has been occurring in recent years, between 2015 and 2019. This enables the introduction of biosimilars that should be much cheaper than the bio-originators [1-3]. Several biosimilars of infliximab, etanercept, adalimum$a b$ and rituximab have been developed. Some of them have already been introduced to the market and there are a lot more to come [1-4].

Yet, it seems that the only reason for using biosimilars instead of bio-originators is the financial pressure [2-5]. There is no medical professional reason for switching a biologic to its biosimilar as biosimilars are not more effective or safer than their originators. In this review, we will briefly discuss the pros and cons of the possibility of switching bio-originators to biosimilars.

\section{The issue of biosimilarity during drug development}

There are certain issues which may cause a problem during the development of biosimilars compared to the originator. Unlike small molecules, biologics are large proteins with a rather complex primary, secondary and tertiary structure. The industrial developmental process has several steps including DNA insertion into the vector, cell culture, fermentation and purification. Even if a very standardized manufacturing procedure is used, two products with the same amino acid sequence may still significantly differ from each other [6]. Moreover, biosimilars undergo "fast development" in order to save money. A full development that includes preclinical, phase I, II and III studies may last for more than 10 years. In contrast, phase II studies are omitted during biosimilar development that would now last for only 7-8 years. Also biosimilars could only be studied in one indication and indication extrapolation can be applied to them $[2,6]$. The issue of indication extrapolation will be discussed later.

On the other hand, if biosimilars and bio-originators are correctly compared in clinical trials, in most cases the high degree of similarity from pharmacological and clinical points of view can indeed be confirmed. For example, in the EGALITY study there were overlapping pharmacokinetic curves between an etanercept biosimilar and the originator [7]. The two molecules also showed very similar efficacy, safety and immunogenicity in psoriasis [7].

\section{The clinician's dilemmas: indication extrapolation, interchangeability and substitution}

As described above, biosimilar studies are usually conducted in one IMID and the results are extrapolated to other diseases. IMID may be similar to each other with respect to pathogenesis. Indeed, all IMID respond to TNF inhibitors. However, recent studies using biologics targeting alternative pathways have indicated that there may be differences even among IMID that may lead to unforeseen consequences regarding efficacy

Address for correspondence:

Zoltán Szekanecz, Division of Rheumatology, Faculty of Medicine, University of Debrecen, 98 Nagyerdei St., 4032 Debrecen, Hungary,

e-mail: szekanecz.zoltan@med.unideb.hu

Submitted: 31.12.2019; Accepted: 8.01.2020 
and/or safety. For example, interleukin 6 (IL-6) inhibitors were effective in RA but not in SpA or psoriasis. On the other hand, IL-17 blockade was effective in SpA and psoriasis but not in RA. Moreover, anti-IL-17 treatment even worsened IBD. Thus, indication extrapolation even within the IMID disease cluster could be a problem when developing biosimilars $[2-4,6]$.

The issue of indication extrapolation may be even more important if that occurs among different disease clusters. For example, the frequency of anti-rituximab antibodies in RA, granulomatous polyangiitis and non-Hodgkin's lymphoma is $11 \%, 23 \%$ and $1.1 \%$, respectively $[4,6]$. Therefore, indication extrapolation among inflammatory and malignant diseases with highly different pathogenesis may lead to immunogenicity and maybe also efficacy and safety issues.

Interchangeability and substitution are two very different terms. Interchangeable biologics, including bio-originators and biosimilars, are expected to have the same clinical profile. Interchangeability is defined by health or regulatory authorities. On the other hand, substitution is when a certain prescribed medicine is replaced by another equivalent drug. This is usually a pharmacist's decision. Automatic substitution of a biologic agent by a pharmacist, at present, is not recommended and it is not allowed in the EU $[4,8]$.

\section{The issue of (multiple) switches}

Certainly the most important issue is whether one can switch from a bio-originator to a biosimilar just because of financial reasons. Again, there is no medical reason for switching an originator molecule to the biosimilar of the same compound [3,4]. It has been suggested by meta-analyses that switching among various anti-TNF agents results in the loss of efficacy after the first, second, third, etc., switches. It has also been shown that switching from one TNF inhibitor to another is still an option, but the third anti-TNF agent may have very little efficacy [4, 9]. Indeed, in our Hungarian cohort, switches to the second, third and fourth treatment resulted in gradual loss of efficacy and decreasing drug survival rates [10]. As a biosimilar is "similar" to its bio-originator but definitely not the same, a forced switch from the bio-originator to the biosimilar of the same compound may be counted as "one more switch" that may, as discussed above, result in loss of efficacy.

On the other hand, in the NOR-SWITCH trial, patients with various IMID were treated with infliximab originator. About half of the patients were switched to the biosimilar at week 52. Patients continuing the originator infliximab and those switched to the biosimilar did not show differences in safety and efficacy [11]. Also, in the
EGALITY trial described above, some psoriasis patients underwent multiple switches between the etanercept originator and biosimilar. Psoriasis Area and Severity Index (PASI) clinical responses were similar between the pooled continued and switched treatment arms [7]. Yet, these were clinical trials in a relatively small set of patients. We still lack a great amount of real-world data [5]. In the real world, it is still unsure whether forced switches performed due to solely financial reasons would result in loss of efficacy or not.

\section{International recommendations}

Finally, in the last published set of European League Against Rheumatism (EULAR) recommendations for the management of rheumatoid arthritis, the Task Force reiterated its position that "if a TNF-inhibitor fails, another TNF-inhibitor - but not a biosimilar of the same molecule! - can be (...) effective. (...) An effective biological agent should not be switched to another bDMARD for non-medical reasons" [12].

Based on these recommendations, the Rheumatology Section of the Hungarian Medical College also proposes that "when choosing a biosimilar product, the following professional and ethical issues need to be considered: a) an effective and safe original biologic agent should not be switched to a biosimilar; b) when initiating biological therapy, a biosimilar alternative may be considered after an agreement with the patient; c) this is primarily an economical consideration so that more patients should have access to treatment" [13].

\section{Conclusions}

The introduction of a biosimilar agent is a good alternative during targeted therapy. It may increase patient access to treatment, widen the choice of health care professionals and may be cost-effective. On the other hand, there are still numerous dilemmas including the possible effects of forced switching on efficacy and safety, issues of automated substitution, open questions in indication extrapolation and a lack of robust real-world data. Although there are increasing biosimilar penetration rates in Northern Europe, we still need more convincing data and real-world experience in our region $[5,8]$.

The author declares no conflict of interest.

\section{References}

1. www.bptc.com.

2. Wilson AW, Neumann PJ. The cost-effectiveness of biopharmaceuticals: a look at the evidence. MAbs 2012; 4: 281-288, DOI: 10.4161/mabs.4.2.18812. 
3. Castañeda-Hernández G, Szekanecz Z, Mysler E, et al. Biopharmaceuticals for rheumatic diseases in Latin America, Europe, Russia, and India: innovators, biosimilars, and intended copies. Joint Bone Spine 2014; 81: 471-477, DOI: 10.1016/j. jbspin.2014.03.019.

4. Sarzi-Puttini P, Marotto D, Caporali R, et al. Biosimilars vs originators: Are they the same? Autoimmun Rev 2019; 18: 102404, DOI: 10.1016/j.autrev.2019.102404.

5. Pentek M, Zrubka Z, Gulacsi L. The Economic Impact of Biosimilars on Chronic Immune-Mediated Inflammatory Diseases. Curr Pharm Des 2017; 23: 6770-6778, DOI: 10.2174/1381612 824666171129193708.

6. Vulto AG, Jaquez OA. The process defines the product: what really matters in biosimilar design and production? Rheumatology (Oxford) 2017; 56 (Suppl 4): iv14-iv29, DOI: 10.1093/ rheumatology/kex278.

7. Griffiths CEM, Thaci D, Gerdes S, et al. The EGALITY study: a confirmatory, randomized, double-blind study comparing the efficacy, safety and immunogenicity of GP2015, a proposed etanercept biosimilar, vs. the originator product in patients with moderate-to-severe chronic plaque-type psoriasis. Br J Dermatol 2017; 176: 928-938, DOI: 10.1111/bjd.15152.

8. Kawalec P, Stawowczyk E, Tesar T, et al. Pricing and reimbursement of biosimilars in Central and Eastern European countries. Front Pharmacol 2017; 8: 288, DOI: 10.3389/ fphar.2017.00288.
9. Solau-Gervais E, Laxenaire N, Cortet B, et al. Lack of efficacy of a third tumour necrosis factor alpha antagonist after failure of a soluble receptor and a monoclonal antibody. Rheumatology (Oxford) 2006; 45: 1121-1124.

10. Brodszky V, Bíró A, Szekanecz Z, et al. Determinants of biological drug survival in rheumatoid arthritis: evidence from a Hungarian rheumatology center over 8 years of retrospective data. Clinicoecon Outcomes Res 2017; 9: 139-147, DOI: 10.2147/ CEOR.S124381.

11.Goll GL, Jorgensen KK, Sexton J, et al. Long-term efficacy and safety of biosimilar infliximab (CT-P13) after switching from originator infliximab: open-label extension of the NOR-SWITCH trial. J Intern Med 2019; 285: 653-669, DOI: 10.1111/joim.12880.

12.Smolen JS, Landewé R, Bijlsma J, et al. EULAR recommendations for the management of rheumatoid arthritis with synthetic and biological disease-modifying antirheumatic drugs: 2016 update. Ann Rheum Dis 2017; 76: 960-977, DOI: 10.1136/annrheumdis-2016-210715.

13. Hodinka L, Bálint P, Bender T, et al. Treatment of arthritides with synthetic and biologic antirheumatic drugs [Hungarian]. Immunology Quarterly (Budapest) 2015; 7: 4-25. 